\title{
TYPOLOGIE DES MARIAGES CHEZ LES MBOCHIS NGAE DU DISTRICT D'ALLEMBÉ
}

ROCK ОКIEMBA Assistant à l'Université Marien Ngouabi rockfeller16@yahoo.fr

RÉSUMÉ : Le but de cet article est d'analyser les caractéristiques des rites du mariage dans la société Mbochi, en République du Congo. En ce qui concerne le travail anthropologique que nous avons effectué dans le sous-groupe ngaé qui se trouve dans la partie septentrionale du pays, et dont les spécificités coutumières du mariage sont l'exigence d'une tradition ancestrale. Cette étude est le fruit d'une enquête orale réalisée dans le district d'Allembé. La chaîne d'alliances matrimoniales réalisée grâce à la compensation matrimoniale appelée «dot» parmi les ngaé, qui ne peuvent avoir des relations sexuelles, sans transgression de la société, exige le respect des valeurs morales. C'est ainsi que la coutume a établi le «mariage» parce qu'il n'y a pas de procréation sans sexualité. Selon cette pratique, il existe différents types de mariage.

MoTS-CLÉS : typologies des mariages; mbochi-ngaé; allembé; Congo.

ABSTRACT: The purpose of this article is to analyse the characteristics of marriage rites in Mbochi society in the Republic of Congo. Regarding the anthropological work that we did in the Ngaé subgroup, whose requirements regarding marriage are part of ancestral tradition. This study is the result of an oral survey carried out in the district of Allembé (Department of Plateaux), on the right bank of the Alima and bordering other subgroups: Téké-limà, Ondinga, Olée, Mbondzi and Ngilima. This Mbochiggae subgroup is found in the northern part of the country. The chain of matrimonial 
alliances achieved through a matrimonial compensation called "dowry" among the Ngaé, who cannot have sex without transgressing society, requires respect for moral values. This is how custom established "marriage" because there is no procreation without sexuality. According to this practice, there are different types of marriage.

KEYWORDS: Typologies of marriages; Mbochi-Ngaé; Allembé; Congo 


\section{Introduction :}

Selon Sautter (1962), l'implantation des mbochi en République du Congo n'est pas ancienne par rapport à son voisin Tégué. Ce groupe se trouve au nord, et est à cheval entre deux départements : la Cuvette et les Plateaux. Chaque département est subdivisé en sous-groupes : Koyo, Akwa, Ngaré, pour la Cuvette ; Olée, Odinga, Tongo, Ngilima, Tsambi-tso, Eboyi, Obaa, Ngaka et Ngaé pour les Plateaux. C'est sur ce dernier sous-groupe que porte notre intérêt, pour connaître son domaine de la parenté nécessaire en anthropologie sociale. C'est ce qu'affirme Fox (cité par Géraud, Lezervoisier et Potier, $2000: 178)$ : «la parenté est en anthropologie ce que la logique est en philosophie et l'étude du nu aux arts plastiques : la discipline de base ».

Cet article repose sur une enquête anthropologique sur les différentes formes de mariages dans la société mbochi ngaé où la multiplicité des lignages qui caractérisent la structure sociale traditionnelle est indépendante les uns des autres. On s'en rend compte, de plus en plus par l'existence des omwè. Ceux -ci sont, par rapport aux lignages, asia, des segments de lignages. Ainsi, baré bà omwè ophoo, "membres d'un même lignage ». Les omwè correspondent sans doute à des lignées. Comme tout groupe de parenté, un omwè est bilatéral. C'est ainsi qu'un homme appartient d'une part, à son omwè patrilatéral et d'autre part à son omwè matrilatéral. Comme les omwè sont inféodés aux lignages, asia. L'ancêtre demeure le même- les lignages ainsi que les lignées constituent pour le groupe mbochi l'armature de la parenté. On peut noter que la survie d'un groupe ne dépend pas seulement de son importance numérique. Larmature psycho-idéologique en est la preuve, aboro alongo, aboro l'ékoma, « la parenté, c'est le lien du sang».

Le mariage peut être défini comme "l'union d'un homme et d'une femme telle que les enfants nés de la femme sont reconnus légitimes par les parents » (Notes and Queries on Anthropology, 6è édition, 1951 : cité par Bonte et Izard, 2002 : 444). Les relations entre personnes dans le mariage ne concernent pas ainsi seulement les conjoints, mais également leurs groupes de parenté, et c'est de l'implication de ces groupes dans le mariage que cette institution tire une légitimité qui la distingue, 
du concubinage. Tambiah et Goody $(1973: 67)$ parlent « des transferts qui vont de la famille du fiancé vers celle de la promise », qu’ils désignent en termes de «prix de la fiancée ». Par contre Spiro (1975:92), tente de donner « une interprétation purement formaliste des prestations de mariage en introduisant l'hypothèse d'une articulation entre « valeur de personnes » et «valeurs de biens ».

Pour constituer une famille, il est nécessaire de passer au rite du mariage. Or dans la société mbochi existe une variété de mariages : quels sont-ils?

Pour répondre à cette question, nous avons recouru à une méthodologie axée sur une enquête anthropologique, essentiellement orale, réalisée en langue èmbosi C25 $5^{1}$ avec l'appui d'un linguiste chercheur ${ }^{2}$, dans un département précis de la République du Congo. Ainsi, sur les onze districts que compte le département des Plateaux, cette étude s'est focalisée sur le district d'Allembé, où réside la plus grande proportion de la communauté mbochi-ngaé qui fait l'objet de notre travail. Pour ce faire, nous avons procédé à un échantillonnage aléatoire, par un tirage sans remise de personnes ressources mbochi-ngaé, tirées des 32 villages du district d'Allembé, et représentant $4 \%$ de la population dudit district (CNSEE, 2009). Ces 32 villages sont subdivisés en deux zones : Yama et Ekassa. Ainsi, en partant de la proportion retenue (environ $4 \%$ de la population), il en découle un tirage de 4 personnes par village, 128 par zones, soit 256 personnes ressources au total pour tout le district.

Ainsi, il nous revient de présenter la typologie des différents mariages tels que observés lors de notre étude dans la communauté mbochi-ngaé.

\section{Le mariage par anticipation}

Cemariageétait unevariante du mariage quel'on nomme ebandiou mbandi. Le vocable ebandi vient du verbe qui, entre autres significations qui sont : « commencer », « débuter », « élire », etc. signifie ici « désigner», « choisir » ou jeter son dévolu sur une femme. En effet ce mariage accordait à un homme, suivant la coutume, le droit de mariage avec un enfant à

I Aire géographique de la langue èmbosi (Embanga Aborobongui, Hamlaoui et Rialland, 2014). 2 Dr Embanga Aborobogui Georges Martial, enseignant chercheur à l'Université Marien Ngouabi. 
naître, surtout dans la perspective d'une fille. Si par exemple une femme était dans un état de grossesse, dzémi, un prétendant s'annonçait pour épouser sa future fille. Au cas où sa candidature était agréée par les parents, celui-ci était tenu, comme à l'accoutumée, de verser à ses futurs beaux-parents les iléli, composés exclusivement en vin de palme. Suivant le désir du futur beau-père, le prétendant pouvait y ajouter en monnaie ancienne, c'est-à-dire les $n z i$, "monnaie en coquillage », ou encore les ngyèlè, trois barrettes servant de même de monnaie ancienne. Il faut dire que faute de connaissances échographiques, ce type de mariage était soumis au gré du hasard des résultats de l'accouchement. A telle enseigne que si le nouveau-né était de sexe féminin, il n'y avait aucun inconvénient quant à la poursuite des démarches conduisant à la concrétisation de l'union matrimoniale. Si par contre le nouveau-né était de sexe masculin, cela était bien désolant pour le prétendant en attente. Dans ce cas, les objets impérissables étaient remboursés au prétendant déçu, sauf le vin de palme. En revanche, des liens d'amitié très durables devaient se nouer entre les deux hommes. Ces liens allaient aussi bien de l'aide matérielle que de l'aide médicale en passant toutefois par la protection physique.

Cette pratique qui du reste paraît étrange avait sans doute ses raisons bien fondées. En premier lieu, elle était révélatrice d'un nombre très insuffisant de femmes à pourvoir. On suppose que ce déficit en femmes pouvait avoir, entre autres causes, l'interdiction bilatérale maximale des mariages endogamiques chez les Mbochi ngaé. En second lieu, le mariage par anticipation avait aussi ce côté qu'on peut qualifier de pédagogique car, dès l'âge de six ou sept ans, le mari retirait la fillette de ses parents pour l'élever selon sa convenance. L'aspect phallocratique n'est pas non plus à écarter dans cette affaire, pas plus que le désir de la déflorer lui-même.

$\mathrm{Du}$ fait de son caractère jugé trop rétrograde, du fait également de l'évolution démographique en faveur du sexe féminin, le mariage par anticipation n'est plus pratiqué de nos jours dans la société mbochi ngaé. Mais en ce qui concerne les 5 autres formes de mariage sont toujours d'actualité. 


\section{Le mariage dit okyénaa}

Avant tout propos, il semble important de donner la signification que recouvre le terme okyénaa. En effet, le substantif okyénaa a pour verbe ikyéna qui vaut dire « couper», "sectionner » Mais okyénaa qui, à vrai dire, décrit le fait de couper revêt ici une connotation sociologique importante.

En fait, okyénaa désigne une union matrimoniale issue d'une rupture provoquée par une femme mariée bénéficiant de la complicité de son nouveau partenaire. Cependant, le terme le plus approprié qui désigne l'acte qui consiste, pour un homme, à arracher de cette façon la femme de l'autre est ipigna. Quand un Mbochi dit ipigni wa mwasi ; il veut ainsi dire, « je lui ai ravi sa femme». Cela dit, on ne saurait imputer la cause de ces mariages aux simples faits des relations conflictuelles époux/épouses, même si ce détail peut paraître important. Si tant est qu'à l'origine les raisons pouvaient être celles-là, il semble que la pratique de ces mariages relevait tout simplement des mœurs propres à la société mbochi d'autrefois. A cet égard, deux cas non moins célèbres de ce type de mariage avaient été notés dans les villages olèmè et poro. Il s'agissait des nommés Ondèè et Mbama-Essongo qui, dans les années 1940-1941 avaient réalisé les mariages okyénaa en payant, chacun pour son cas, la surenchère dotale comprise, la somme de $500 \mathrm{~F} \mathrm{CFA}^{3}$. Comme l'a justement souligné Soussa (1981 : 155) : «ce mariage dit okyénaa revêt la forme d'une succession de défis et de ripostes; défi lancé par le prétendant contre l'ancien mari qui réclame le remboursement illico de son obwè... ». De cette façon, on pense que le mariage okyénaa prenait la forme d'un défi économique dans lequel la richesse, seule garantie dans cette affaire, devenait ostentation pour qui arrachait à l'autre sa femme, puisque le dédommagement du mari frustré devait se payer au double et sur-le-champ.

Le nouveau partenaire ne remettait pas cette somme directement à l'ancien mari. Cette somme transitait par le père de la femme.

Les facteurs matériels associés à l'esprit de défi étant nécessaires pour la réalisation d'un tel mariage, on n'était pas étonné d'apprendre que le

3 Communauté Financière Africaine, regroupant les pays d'Afrique centrale et de l'Ouest, principalement les anciennes colonies françaises, utilisant cette monnaie dont la parité à l'euro est : 1 euro $=655 \mathrm{~F} \mathrm{CFA}$. 
mariage okyénaa profitait aux adultes, mariés ou non, et non aux jeunes célibataires qui, eux, fautes de moyens matériels et financiers leur donnant libre accès aux femmes, se voyaient incapables de se lancer dans cette entreprise périlleuse.

\section{Le mariage dit okwélé}

Ici aussi, le terme okwélé mérite d'être éclairé. Le vocable okwélé vient du radical kwélé qui désigne tous les biens, humains et matériels, laissés par le défunt, owé. Il convient de préciser que mot kwélé est en usage chez les Mbochi des variantes ngaé et mbondzi. Chez les Mbochi dit Olée, ceux dont il est plus question ici, on emploie à la place le mot isèè.

Pour tout dire, okwélé rend compte, en fait, de la situation où une ou plusieurs femmes survivantes observent strictement un deuil à la mémoire de leur défunt mari. Ainsi donc okwélé désigne à proprement parler, à la fois, le «veuvage » et le « deuil».

Okwélé est un terme discriminatoire ; il discrimine en cela les femmes. La langue èmbosi permet de dire, ayiri ba kwélé, «les femmes veuves ». Mais dire abaa ba kwélé, "les hommes veufs " s'avère un non-sens ; l'inverse n'est pas possible. Ce système de pensée qui exclut le veuvage dans le cas d'un mari survivant est complètement absent aussi bien de la langue que de la structure mentale. Ainsi qu'expliquent les Mbochi, si le veuvage n'est pas pensé en terme masculin, c'est parce qu'un mari survivant ne faisait jamais l'objet d'un bien qu'on devrait hériter, tel est le cas d'une veuve.

Comme parmi les biens laissés par un owé, « un défunt », les veuves ou la veuve étaient d'un enjeu important le jour de l'adjudication de l'héritage, essayons de montrer en quoi consistait ce mariage dit okwélé.

Ainsi pour le remariage de la veuve, l'option matrimoniale sur elle était accordée à toute la parenté survivante du mari défunt. Pour ce faire, les héritiers présomptifs étaient classés suivant l'ordre généalogique. En premier lieu c'étaient les frères cadets du défunt. En second lieu les neveux utérins, bana ba abola.

La cession de la veuve aux ayants-droit en compétition ne pouvait pas se faire sans que son avis fût sollicité au préalable. Ce qui est vraisemblable, c'est que celui qui a été choisi est, comme on l'a couramment 
constaté, celui avec qui elle était en complicité amoureuse du vivant de son mari. Il fallait qu'il fût parmi les parents désignés dans les deux premiers groupes. S'il n'était pas de ceux-là, ses droits étant toujours reconnus comme membre faisant partie du lignage, il se devait de dédommager les ayants-droit frustrés en leur offrant du vin de palme plus un objet de valeur en guise de compensation matrimoniale. Par cet acte, les Mbochi disent de l'époux-héritier qu'il a « acheté la veuve »; asombi oyiri ya kwélé.

Ce geste, plus symbolique encore que l'est la vraie compensation matrimoniale avait tout l'air d'un rituel. On l'appelait, lémbindi la okwélé. Le premier terme, lémbindi, qui a un lien étroit avec mbindi, signifie « la souillure »; la okwélé, « du veuvage ». Présenté dans le folklore mbochi comme un rituel, lémbindi la okwélé n'était rien d'autre qu'un rite purificateur. En cela, il retirait à oyiri ya kwélé le deuil porté. On peut ajouter que cette pratique correspondait en réalité à un rite de réintégration sociale. De cette façon, celle qui était veuve pouvait sans restriction aucune participer à la vie sociale et économique, continuer à reproduire au bénéfice de la belle-famille en général et à l'époux-héritier en particulier.

Il est nécessaire de rappeler - car ceci est important - que le mariage dit okwélé ne pouvait pas se réaliser sans que les intérêts de la parenté paternelle et la parenté maternelle ne furent confrontés. Ainsi, lorsqu'il n'y avait qu'une seule veuve à pourvoir, celle-ci était de droit échue au groupe des paternels, la raison étant que cette femme avait été épousée grâce aux biens de la « dot » fournis par le père du décédé. Au cas où l'époux-héritier était un frère ou cousin de la parenté maternelle, le dédommagement dont nous avons parlé allait impérativement s'imposer.

Par contre, si le défunt était polygame, la première veuve était en priorité cédée aux agnats, (aux paternels), la seconde aux utérins, (aux maternels).

Cela dit, l'héritage de la veuve, qui se confond ici avec le mariage avec celle-ci, mariage dit okwélé, ne saurait être assimilé au lévirat qui, suivant l'honorable sens qui lui est conféré, fait du lévir celui qui engendre une descendance posthume dont il n'est que le substitut du père.

Ainsi qu'on a pu le constater, la situation précédemment décrite indique en toute clarté qu'il n'y a de mariage dit okwélé que lorsque la ques- 
tion du remariage mieux de l'héritage de la veuve se résout dans un cadre strictement familial.

En revanche, si oyiri ya kwélé, la veuve, avait par exemple retenu des griefs contre les membres survivants de la parenté de son défunt mari, (sorcellerie, malveillance, méchanceté ou quelque autre motif, etc.), pour ne pas en épouser un seul, toute sa parenté était tenue de réparer le préjudice causé pour avoir rompu le lien conjugal avec les parents du défunt mari. A cause de cela, la compensation matrimoniale devait être remboursée. Mais pour échapper à cette amende, l'astuce pour les veuves est de dire, «je reste dans la famille de mon mari sous couvert de mes enfants... ", en choisissant notamment l'aîné des garçons comme « époux » formel.

A vrai dire, les cas où les veuves se refusaient à prendre époux parmi les parents survivants de son défunt mari étaient rarissimes dans le contexte de la vie paysanne. Il fallait, pour celles-ci, rester dans le groupe de ses beaux-parents pour préserver les enfants des attaques en sorcellerie. Ce fait est illustré par le dicton suivant : " avec sa queue, le singe ne peut pas en toute assurance passer au-dessus d'un feu... », le risque étant de se faire enflammer la queue qu'il traîne. D'une façon imagée, le singe est ici la veuve et la queue, les enfants. Cela en réalité implique que si le fait pour une veuve de quitter ses beaux-parents est réalisable, le faire avec les enfants serait à tous les coups une entreprise périlleuse pour ceux-ci.

Ainsi, tout laisse penser que dans la société mbochi, le mariage dit okwélé apparaît comme une nécessité aussi bien pour la veuve que pour les beaux-parents de celle-ci de consolider le lien conjugal que même la mort ne saurait briser.

\section{Le mariage avec la sœur de l'épouse}

$\mathrm{Au}$ point de vue linguistique, nous déplorons ici l'absence dans la langue èmbosi, d'un terme générique ou descriptif traduisant cette forme d'union matrimoniale.

Le mariage d'un homme avec notamment la sœur cadette de l'épouse était d'un usage très courant dans la société traditionnelle mbochi. C'est ce qui est nommé le sororat ou encore le mariage sororatique. 
Pour que celui-ci fût possible, il fallait qu'un homme se trouvât dans des conditions particulières qui étaient les suivantes ; stérilité de l'épouse, décès de celle-ci, etc.

Cependant, le manque de maternité chez une femme ne menaçait guère la stabilité de la vie conjugale. Mieux, elle accordait - tel que la coutume le prescrit - à son mari une option matrimoniale sur ses sœurs cadettes directes ou indirectes. Reste que la prééminence était accordée aux sœurs cadettes directes. A défaut de celles-ci, on puisait alors dans le rang des cadettes indirectes une jeune fille apte à assumer des tâches ainsi dévolues à une femme. La fille cédée n'avait plus droit à un versement de la « dot » pleine ; celle-ci ayant été déjà versée lors du mariage de son aînée. Mais dans l'intérêt de légitimer sa seconde union, l'homme se devait, d'une manière toute symbolique, d'offrir à ses beaux-parents quelques biens n'excédant pas les montants exigés de coutume.

A propos toujours de la stérilité d'une femme, une expression ainsi consacrée traduisait le fait, pour la femme stérile, de s'être adjointe une sœur comme coépouse. On disait d'elle, asi kana ngwanga. Ce qui signifie, «elle a mis ngwanga». Or le terme ngwanga traduit «la servitude». On est donc en droit de supposer que la femme a désigné « celle qui lave les défauts » ou « celle qui répare les fautes », ce qui s'entend, de sa sœur aînée.

En revanche, quand il s'agissait de remplacer une sœur aînée décédée, la sœur remplaçante n'était plus ngwanga. Elle était considérée au même titre qu'une épouse légitime. Les enfants nés d'elle et ceux nés de la sœur ainée décédée avec le même époux l'appelaient tous, en termes d'adresse, niongo ; en termes de référence, ngoo; sans aucune discrimination.

$\mathrm{Au}$ fait, l'introduction d'une ngwanga dans un ménage à la volonté d'une femme souffrant d'un handicap était justificative de bien de choses. Premièrement, la ngwanga était l'alliée sinon la complice inconditionnelle de son aînée. Au cas où cette dernière était en conflit avec son époux ou menaçait de le quitter, elle bénéficiait ainsi de la solidarité de sa cadette. Pour l'homme, plutôt que de perdre une seule femme, il les perd toutes les deux.

Deuxièmement, la ngwanga servait par ailleurs de prétexte à une femme mariée dont les intentions, avouées bien sûr, étaient de faire pro- 
fiter les avantages matériels de son foyer conjugal à sa seule famille, ce qui était profitable à la polygynie entre les sœurs.

Pour tout dire, le sororat, qui est le mariage d'un homme, veuf ou pas, avec la sœur cadette de sa femme et le lévirat, qui est le mariage d'une veuve avec le frère cadet de son défunt mari sont, dans tous les cas, appelés « les mariages préférentiels », en tant qu’ils sont basés sur la préférence des conjoints. Qui plus est, le lévirat et le sororat, dont la diffusion fut et reste si étendue, ont pour cause une ancienne conception du mariage comme un contrat entre groupes plutôt qu'entre individus.

Etant donné l'intérêt que suscite en nous l'étude des mariages préférentiels, il est nécessaire qu'on se penche, dans les lignes qui vont suivre, sur un mariage de même type que le précédent.

\section{Le mariage avec la fille du frère de l'épouse}

Dans le cas précédent tout comme dans celui-ci, on constate une fois de plus l'absence d'un terme local désignant le mariage dont il est question ici. Pour pallier ce manquement, les Mbochi caractérisent ce type de mariage de iba alongi. Mot à mot, cela se traduit ainsi ; iba, «le mariage »; alongi, participe passé du verbe ilongoso qui signifie " parfaire ». En clair, iba alongi veut dire "mariage réussi », "mariage parfait », " mariage de succès ", etc. D'une manière plus explicative, cette expression est utilisée pour illustrer le fait que le mariage d'un homme avec la fille du frère de sa femme n'était possible que dans le ménage où la réussite, la vie en bonne intelligence, etc. servaient de garanties pour que la tante paternelle cohabitât avec sa nièce qui est la fille de son frère comme des coépouses.

Ce type de mariage qui accordait à un homme un droit matrimonial sur la nièce paternelle de sa femme était, autant que le sororat, d'un usage très courant dans tout le pays mbochi, plus particulièrement chez les Mbochi dit Oléé et ceux dit Ondinga, tous du département administratif des Plateaux, partie septentrionale du Congo.

A la différence du mariage précédemment décrit, la réalisation de ce mariage-ci n'était pas nécessairement justifiée par la stérilité encore moins par le décès d'une femme à qui il fallait absolument trouver une 
remplaçante au sein de sa propre famille. En voici un exemple parmi tant d'autres.

Une femme, aurait-elle constaté le surplus de travail (entretien des enfants très jeunes, activités champêtres et domestiques) etc. qu'elle sollicitait de l'aide auprès de son frère qui devait lui confier sa fille. Celle-ci devait vivre aux bons soins de sa tante paternelle sa vie durant.

Si la jeune fille se faisait distinguer par ses capacités de travail condition du reste essentielle - sa tante faisait à son mari une proposition allant dans le sens d'amener ce dernier à s'adjoindre, pour épouse secondaire, cette nièce. Au cas où son mari agréait cette proposition, sa femme se rendait chez son frère pour l'informer.

La coutume de céder en mariage sa fille au mari de sa sœur étant courante dans cette société, très peu d'hommes d'ailleurs n'y trouvaient aucun inconvénient. Ce qui effectivement ne faisait que contenter le père de la jeune fille qui trouvait là une occasion toute faite pour recevoir de son beau-frère quelques litres de vin de palme, olèngè ; on ne pouvait, dans ce cas-ci, parler d'un obwè plein puisque celui qui avait été donné lors du mariage de la tante paternelle suffisait à accorder au preneur de la jeune fille un droit matrimonial aussi bien sur les sœurs cadettes que sur les nièces de la femme.

Seulement, si l'initiative d'épouser la nièce paternelle de la femme venait de l'homme lui-même, l'obwè lui était réclamé, payable suivant les exigences de la coutume.

Le cas où un homme recevait de la part de sa femme une épouse supplémentaire sans verser la « dot » ou l'obwè, correspond, pour l'homme, à ce qu'il convient d'appeler « droit d'appropriation ». Dans l'autre cas, où un homme contracte une union préférentielle avec dot, c'est un « droit de préemption ».

Comme dans le cas du sororat, cette nièce était aussi considérée comme une ngwanga, "celle qui était venue laver la vaisselle», "celle qui était venue garder les enfants...».

Cela dit, ce type de mariage aurait pu paraître incestueux dès lors que la tante qui est « le père féminin », tasamwaré, mélange avec sa nièce qui est sa «fille » de même sang, la même semence qui est du même mari. 
C'est ce qu'on appelle «l'inceste de deuxième type ». Ce qui n'est pas du tout le cas ici car on a le sentiment que les Mbochi privilégiaient plus les rapports matrimoniaux que les rapports parentaux.

Les anthropologues ont qualifié ce type de mariage de "mariage oblique », c'est-à-dire du mariage qui unit deux individus de générations alternes, dans le cadre de ces mariages dit préférentiels. Par exemple, le mariage d'un homme avec la veuve du frère de sa mère, avec la fille du frère de sa femme, avec la veuve de son père sauf sa propre mère, avec la veuve du père de son père sauf sa grand'mère paternelle.

En tous les cas, ce type de mariage préférentiel n'est pas sans conséquence sur la famille d'origine, plus exactement sur la filiation. Il conduit à ce que deux individus issus d'un même père puissent se trouver dans la double relation de frère ou de sœur, en même temps de père et de fils d'un côté, de frère de même père et en même temps d'oncle maternel à nièce ou neveu, de l'autre côté.

Il importe de signaler, pour conclure, que les mariages préférentiels chez les Mbochi, tel que nous venons de les présenter, ont pesé de tout leur poids sur la structure sociale. Les liens de parenté, extensibles et communs à presque tous les Mbochi, sont très certainement la conséquence de ces mariages.

\section{La polygynie ou polygamie}

La polygynie est un système social reconnaissant le mariage d'un homme avec plusieurs épouses.

Moro onè o baré. Ce proverbe, du reste incitatif, traduit par son contenu le désir de procréer. Celui-ci constitue pour la société l'élément capital de l'existence sociale. On peut littéralement le traduire en ces termes : " homme grand grâce des gens ». Suivant la vraie signification, ce proverbe veut tout simplement dire « qu'un homme n'avait de grandeur dans la société que s'il possédait un nombre très important de descendants... ». Ceux-ci constituaient sa richesse principale, okulu. Si bien qu'à sa mort, pour ce qui allait composer son legs, ses femmes ainsi que ses enfants avaient plus d'importance qu'autre chose. La société mbochi dans son ensemble témoignait plus de sympathie à l'égard d'un tel homme dont le 
nombre assez considérable d'enfants était d'un apport démographique non négligeable.

De même, la société mbochi savait gré aux femmes prolifiques. On disait de ces femmes : oyiri wo la abora, « cette femme possède des maternités ».

Procréer pour un homme signifiait aussi qu'il fallait à tout prix perpétuer la descendance. Ainsi, un homme pourvu d'une suite importante se voyait déjà, à sa mort, promu généalogiquement au rang des ancêtres, mieux les ancêtres éponymes. C'est pourquoi il est aisé de comprendre l'indifférence de la société à l'égard d'un homme stérile, léngégna ou èyèbhè. En effet l'homme stérile était dans cette société comparable à un marginal. Mieux il était traité en paria. A cause de ce handicap, la fonction de chef de village par exemple lui était inaccessible.

A l'intérêt de beaucoup procréer s'ajoute, comme l'une des causes de la polygynie, l'abstinence sexuelle chez l'homme dont la femme est en train de connaître la période des menstrues, ita la swéngé, "voir la lune », d'une part, et la période correspondant à l'allaitement, d'autre part, d'un nouveau-né.

De tradition, lorsqu'une femme voyait ses règles, son mari s'interdisait d'avoir avec elle des rapports sexuels pour des raisons tenant à l'hygiène car il fallait, surtout pour l'homme qui travaillait dans le domaine magico-religieux, éviter la souillure. L'interdit le plus strict s'imposait à l'homme dont la femme était en période de grossesse, période qui allait se prolonger jusqu'à celle de l'allaitement du nouveau-né. Cette période pouvait couvrir deux ans jusqu'au sevrage de l'enfant.

Malheur à cet homme-là qui pouvait violer cet interdit car, en cas de maladie infantile, surtout les diarrhées répétées, ignaa, une certaine morbidité, etc. il en était tenu inexorablement pour responsable, puisque dans leur imaginaire collectif, les Mbochi tiennent pour établi, que le mélange du sperme, alémi, du père avec le lait maternel, abè, constitue une solution infecte, nocive, pour la santé de l'enfant. Le voisinage disait, en ce qui concerne l'incident provoqué : akiéni mwana ondèmbè ; «ils (le mari et la femme) ont coupé l'enfant précocement. Disons que c'est plutôt 
par association d'idées que par une réelle connaissance des phénomènes chimico-biologiques que les Mbochi entretiennent une telle croyance.

Un autre fait est que par le biais de la polygynie, l'homme condamné à observer le tabou des rapports sexuels pendant les périodes citées contournait ainsi une difficulté de nature judiciaire et économique qui est l'adultère. Car celui-ci est punissable et qui en était puni était mis à l'amende, donc au payement des objets en nature ou en espèces au mari victime.

A ces diverses causes que nous continuons d'énumérer, il importe d'ajouter, en second lieu, l'infécondité de la femme. La femme inféconde est nommée, ekomba. Pour pallier ce handicap, des soins traditionnels lui sont administrés par un thérapeute nommé, nganga ya abora; nganga, «devin-thérapeute»; ya «de»; abora, «les maternités ou les naissances». Dans leur imaginaire collectif, les Mbochi pensent que cette stérilité a toujours eu pour cause l'œuvre d'une entité malfaisante, d'où le recours à celui qui soigne en même temps le corps et l'esprit (Daho, 1983 ; Itoua, 2007).

Entre autres causes, on peut évoquer aussi celle liée à l'inconduite ou à la paresse de la femme. Dans l'intérêt de conserver son prestige social du fait du nombre croissant d'épouses, un homme se refusait à rompre son union conjugale avec son épouse malgré les défauts cités, sauf le vol.

Les naissances considérées anormales (enfants morbides, infirmes), etc. avec une femme infléchissaient aussi la tendance d'un homme quant à s'offrir une épouse supplémentaire. Dans ce cas-ci comme dans celui de la stérilité féminine, les causes étaient imputables à la sorcellerie, alors que les données hématologiques pouvaient en être les causes.

Des causes toujours de la polygynie, il convient de citer en dernier cas celui relatif au prestige social dont jouissait l'homme polygyne dans la société. En fait, le nombre croissant d'épouses était autrefois le critérium de la richesse d'un homme. Il est important, cependant, de souligner que les hommes les mieux placés dans cette entreprise étaient souvent les chefs couronnés en même temps patriarches des groupes domestiques. C'est à croire que la polygynie en pays mbochi était l'apanage des hommes âgés, c'est-à-dire des adultes, des vieux et surtout des chefs. Pour justifier un 
tel fait, l'explication - qui du reste peu paraître un prétexte - était que ceux-ci, imbus d'expérience de la vie sociale entretiendraient les femmes mieux que les jeunes.

Du fait de l'autorité qu'ils incarnaient au sein de leurs groupes domestiques, ces chefs et patriarches polygynes avaient autour d'eux plusieurs dépendants : frères cadets, fils, petits-fils, neveux agnatiques (fils des frères), rarement neveux utérins (fils des sœurs) auxquels ils pouvaient, le cas échéant, redistribuer quelques-unes de leurs épouses.

Il va sans dire que l'accumulation des épouses était surtout bénéfique pour le polygyne qui voulait s'enrichir économiquement. La raison était que dans cette société paysanne où l'activité primordiale était l'agriculture, les femmes, les enfants et les autres dépendants constituaient pour le polygyne patriarche une main-d'œuvre pour ainsi dire corvéable. Une expression mbochi sous-tend d'ailleurs cette réalité : okulu baré ; littéralement, «la richesse, les hommes »; mais au sens profond du terme, cela signifie que «seuls les hommes sont créateurs de richesse »; par extension, « les hommes ont valeur de richesse».

Comme nous venons de le voir, l'énumération des diverses causes de la polygynie nous a permis de saisir les conditions ainsi que le contexte socio-économique dans lesquels un homme était contraint de s'adjoindre des épouses supplémentaires. Ceci nous conduit dans le droit fil de l'idée exprimée par Zambo Belinga (2005 :22), quand il dit :

L'homme, autrefois, ne devenait pas spontanément polygame. Il se préparait au contraire à n'épouser qu'une seule femme. Il ne devenait polygame que beaucoup plus tard, à la suite de certaines circonstances ou sous l'impératif des raisons étrangères à sa volonté.

Par contre, il est des situations qui, au lieu d'être à l'origine de la polygynie n'ont au contraire fait que la renforcer. Ainsi en est-il des mariages secondaires (mariages avec la sœur cadette de l'épouse, mariages avec la fille du frère de la femme) etc., des mariages obliques (mariages avec la veuve $d u$ père de la mère ou $d u$ père $d u$ père, mariages avec la veuve du père, mariages avec la veuve du frère de la mère), etc. qu'on peut encore appeler des « remariages pour cause de veuvage ». A ce sujet, il serait amusant de citer en exemple le cas - encore récent - d'un rusé 
jeune homme, habitant le village Otsiini et qui, parâtt-il, était parvenu à construire un ménage polygyne avec ses six femmes toutes acquises en héritage de ses aînés défunts dont il était, pour chacun d'eux, un héritier potentiel. Signalons que dans cette affaire, la ruse consistait pour ce jeune homme à contracter des unions sans frais en évitant le versement des « dots » comme l'exige la coutume pour chacune de ces femmes.

Cela dit, le nombre de femmes chez les Mbochi n'était pas limité, ainsi l'a confirmé Soret (1954). Pour le cas d'espèce, on peut citer le patriarche Ibara Mbémbé du village Ngagnia qui, à lui seul, avait pu totaliser pas moins de cinquante femmes, un record jamais égalé dans tout le pays mbochi.

Agé de cent ans environ à sa mort en 1982, ce puissant patriarche a laissé pas moins de cent enfants. On peut déjà imaginer le nombre très croissant des petits-enfants et arrière-petits-enfants. Aux dires de ses voisins et autres membres de sa famille, sa descendance était nombreuse au point qu'il n'arrivait pas lui-même à reconnaître certains de ses enfants. Lorsqu'il lui arrivait d'avoir un certain contrôle sur certaines de ses épouses, Ibara Mbémbé avait comme la magie, à chaque naissance qui survenait dans son ménage, de reconnaître ceux des enfants qui, biologiquement, n'étaient pas issus de lui. Ce sentiment permanent de douter quelquefois de la filiation consanguine d'un enfant venait du fait que nanti de toutes ses épouses citées, il devait, en certaines occasions, se sentir à bout de ses capacités tant physique que morale pour procréer ou maintenir la cadence normale de ses rapports sexuels. On dit toujours qu'un enfant qu'il supposait n'être pas le sien était appelé à trépasser les jours suivant sa naissance ; ceci, sous l'effet des imprécations prononcées par lui. N'était-ce pas cela plus un mythe qu'une réalité?

Comme pour le cas du jeune rusé d'Otsiini, Ibara Mbémbé avait lui aussi bénéficié des veuves laissées par son père polygyne, chef de Terre, de celles laissées par ses oncles maternels en sa qualité d'héritier toujours. Ces veuves en mariages secondaires, ajoutées aux épouses en mariages primaires avaient $\mathrm{pu}$, de toute évidence, enrichir son harem. 
Cette pratique montre profondément que les mariages pour cause de veuvage ou mariages d'héritage ne faisaient qu'assurer la pérennité du système polygynique.

Au reste, la vie domestique d'une union polygyne chez les mbochi prenait en considération, pour son fonctionnement, trois dimensions qui étaient celles des rapports mères/enfants, époux/épouses et enfin, les épouses entre elles.

Comme chaque épouse disposait d'une case-cuisine, la relation biologique mère/enfants semblait se renforcer sous cet aspect, tous les enfants issus d'une même mère se regroupant autour de celle-ci aux fins de recevoir des soins nécessaires à leur éducation. Autant dire que la structure des unions polygynes reposait sur des familles matricentrées.

En général, le bon sens obligeait une femme à considérer les enfants de sa coépouse comme les siens au point de leur apporter toute l'assistance nécessaire. Mais en particulier, le sentiment de jalousie entre cette femme et les enfants de sa coépouse était quelquefois prévisible. Souvent, ce sentiment de jalousie, illustré par le manque de tendresse vis-àvis de l'enfant de l'autre, avait un lien avec la querelle entre mères. D'une façon globale, on peut avouer que de tels faits étaient minimes au regard de la parfaite entente qui avait toujours régné dans une famille polygyne mbochi entre mères et enfants différents.

Dans cette société traditionnelle, le mari exerçait un pouvoir sur ses femmes même s'il était ou non soumis lui-même à l'autorité d'un autre homme qui était son aîné. Mais un homme marié, vivant avec ses femmes auprès d'un aîné (comme cela était courant), voyait son autorité diminuée $\mathrm{du}$ fait de cette cohabitation. L'aîné en question régentait toute l'autorité domestique. Les femmes d'un tel homme étaient soumises à la fois à l'autorité de cet aîné et à celle de leur mari, pour la raison que dans cette société paysanne, le mari ne pouvait pas faire face aux problèmes du logement et de la propriété de sol à cultivable sans passer par son aîné.

Si le mari n'était pas soumis à l'autorité d'un aîné, il se trouvait luimême, dans ses rapports avec ses femmes, dans la situation d'un aîné. Ainsi, il attendait de chacune d'elles de la nourriture qu'il consommait avec certains parents plus jeunes, en tout cas sous son toit. Inversement 
celles-ci devaient attendre de lui assistance et surtout protection. A ce sujet, la tâche du mari était souvent pénible. Il devait veiller à la bonne santé de ses épouses, fournir du bois de cuisson, veillé à ce que le stère de bois appartenant à chaque épouse ne s'épuise, procéder, si possible, à la réparation de chaque case-cuisine.

Entre autres obligations, on peut signaler ses activités sexuelles avec chaque épouse. En effet le mari polygyne réglait toujours ses activités sexuelles suivant un calendrier établi par lui-même. A l'accoutumée, c'était tous les deux jours qu'il devait passer la nuit avec chaque épouse. $\mathrm{Au}$ cas où il ne respectait pas ce rythme et qu'une épouse s'en sentait lésée, cela était le motif palpable d'une brouille entre lui et cette épouse.

Limprudence causée par le mari pour un séjour plus long chez une femme particulière au détriment des autres femmes était aussi un fait générateur de discordes entre femmes. Mais la forte autorité du mari permettait d'éviter, éventuellement, des conséquences fâcheuses : empoignades, insultes, etc.

Signalons que le manque de rapports sexuels chez la femme n'incitait pas pour autant celle-ci à commettre l'adultère. C'est ce qui amenait les hommes à honorer « la fidélité » dont faisaient montre les femmes mariées d'autrefois. Ils ignoraient ou faisaient semblant d'ignorer que du fait de la coutume qui autorisait les rapports sexuels en toute impunité entre une femme mariée et les dépendants de son mari : neveux utérins, frères cadets, pour ne citer que ceux-là, cette femme trouvait là l'occasion, non seulement d'échapper à la sanction pour adultère, mais aussi de soulager ce désir qui l'accablait tant.

Même si les devoirs d'un époux polygyne étaient équitables envers toutes ses épouses, l'affection ainsi que la préférence pour l'une ou l'autre des épouses étaient tout autre chose. En clair, les sentiments amoureux d'un époux polygyne n'étaient en tous les cas point uniformes à l'égard de toutes ses épouses. C'est pourquoi, parmi celles-ci, on distinguait toujours celle qui était appelée, kuéndé, ou mwasi ya kuéndé, «la préférée » ou « la femme préférée », celle à l'égard de qui le mari portait plus d'attention. Dans bien de cas, il s'agissait de la plus jeune des femmes, la dernière arrivée. Sur ce cas il y a discussion car la femme kuéndé peut aussi 
être la première femme, notamment la plus âgée de toutes. Les critères qui déterminent cette préférence restent toutefois inexplicables chez nombre de maris polygynes. En tout état de cause, une femme est kuéndé suivant les caprices voire la volonté du mari polygyne lui-même.

En ce qui concerne les rapports entre épouses, celles-ci vivent entre elles dans les rapports de abia ba ika; abia, "amis », " camarades »; ba, «de»; ika (plur.), oka (sing.), « rivales ». Si le terme générique oka signifie bien « rivale », on est en droit d'inférer que leurs rapports sont naturellement des rapports de rivalité mieux des rapports qui n'excluent pas les conflits.

Même si cela semble au fond vrai, il faut cependant avouer que de coutume l'esprit de camaraderie, de collaboration et d'entraide animait les rapports entre rivales. C'est pourquoi dans leur vie au quotidien, les plus jeunes coépouses honoraient les plus âgées en les nommant, tomi, « chère aînée ».

Cela dit, il n'est pas erroné d'affirmer que l'atmosphère de paix qui dominait les rapports entre rivales dans la totalité des ménages polygynes du pays mbochi avait pour explications :

- Le caractère institutionnel, social et culturel de la polygynie,

- La séparation individuelle des habitations des épouses (ce qui en réalité excluait la promiscuité, source de tensions psychologiques dans bien de cas),

- La coopération économique obligeant le travail en groupe sur un même domaine ;

- Enfin et surtout, la très forte autorité du mari sans oublier sa volonté d'être quitte dans ses obligations envers toutes ses épouses, etc.

Ajoutons par ailleurs que, ce qui du reste contribuait à l'amélioration des rapports entre coépouses, c'était le fait que de coutume, la première épouse voyait toujours de bon œil la venue au foyer d'une autre femme à cause du surtravail domestique imposé par le nombre non moins important des dépendants du mari qu'il fallait régulièrement nourrir.

Il arrivait aussi que dans cet intérêt de se partager les lourdes charges domestiques, la première épouse pût imposer sa sœur cadette à son mari 
parmi ses autres coépouses ; on était là en face d'une polygynie sororale, c'est-à-dire d'une polygynie où les coépouses sont des sœurs, soit directes soit indirectes telles les cousines. Ce type de polygynie sororale où chaque épouse avait tendance à céder à son époux sa sœur cadette montre qu'il s'agissait là d'une polygynie autocentrée horizontalement, c'est-à-dire d'une union où les relations entre sœurs pivotaient autour de la sœur aînée.

Il va sans dire que la polygynie sororale comporte autant de bienfaits que de méfaits. En ce sens que si une épouse était docile, soumise, attentionnée, aimable à l'égard de son mari, sa sœur cadette l'était également. Si par contre elle était de mauvaise conduite et pis, si elle menaçait de partir du foyer conjugal, sa sœur cadette la suivait irrémédiablement. Ce dernier aspect est, autant qu'on pense, celui des plus néfastes de la polygynie sororale. Sinon, les seuls faits positifs d'une union polygyne sororale résident, pour l'homme polygyne, dans l'absence de conflits entre épouses. Et pour celles-ci, dans l'esprit de collaboration sincère, de relations naturellement amicales voire de complicité. Pour les enfants, dans la solidarité des demi-germains par le père et de frères et sœurs par leurs mères.

Le regard tant soit peu exhaustif que nous avons jeté sur l'organisation interne de la famille polygyne mbochi conduit à la conclusion suivante.

Nous avons en effet remarqué au sein de ce type de famille trois aspects essentiels.

$\mathrm{Au}$ premier aspect apparaissait la dimension hiérarchique des rapports époux/épouses qui, elle-même, suivant les cas, dépendait ou non de la hiérarchie époux/aîné de l'époux. Il faut dire que même dans le cas où le mari polygyne cohabitait avec son aîné, cette hiérarchie n'excluait pas pour autant l'autorité incontestable du mari lui-même vis-à-vis de ses épouses. Sauf que pour les grandes décisions à prendre contre l'une ou l'autre des épouses, par exemple la séparation de corps ou la tentative de divorce, etc., le dernier verdict émanait de droit de cet aîné.

En ce qui concerne les rapports mères/enfants, il importe de noter que dans ceux-ci, l'autorité du père sur tous les enfants basculait en grande partie celle des mères, malgré la relation naturelle mère/enfant qui a tou- 
jours été forte et malgré aussi la promiscuité imposée par la cohabitation d'une mère avec ses enfants dans une même case-cuisine.

Notons cependant qu'une telle cohabitation avait pour impact la tendance de chaque mère à se sentir affectueusement plus près de ses propres enfants, sans omettre, chez ceux-ci, la solidarité des germains. On peut à ce propos supposer que même si d'habitude les bons sentiments d'affection d'une mère quelconque s'étendaient sur tous les autres enfants, cela, à vrai dire, restait lié aux apparences. Car en réalité cette situation ne manquait pas de voiler une certaine discrimination affective.

Le dernier aspect qu'il convient de soulever est celui des rapports entre coépouses, ika. Le principal enseignement qu'on peut en tirer est qu'en principe il n'était jamais établi, dans la société mbochi, une hiérarchie entre les épouses d'un homme suivant leur ordre d'arrivée au foyer. Être la première femme d'un homme n'impliquait nullement d'être la mấtresse de maison, pas plus que cela ne donnait d'autorité sur les autres épouses. Seulement, du fait de son expérience et de sa crédibilité auprès de ses coépouses, celles-ci, d'une façon implicite, lui reconnaissaient toutefois la primauté des rôles domestiques.

\section{Conclusion}

Cette étude que nous avons menée dans la circonscription du district d'Allembé, nous a permis de comprendre dans une certaine mesure les typologies des mariages chez les Mbochi Ngaé. Pour l'anthropologie mbochi, ne peut avoir un enfant qu'un homme qui a payé sa compensation matrimoniale. Une sorte de contrat explicite qui ne dit pas son nom dans cette société. C'est ce qu'affirme Fouillée (1880:115) : «Nous naissons dans une société déterminée, et cela malgré nous, sans doute nous acceptons ensuite le fait accompli, et quand nous arrivons à l'âge de la majorité, nous adhérons par nos actes même au contrat social en vivant au sein de l'Etat et sous les lois communes ». 


\section{Sources orales}

\begin{tabular}{|c|c|c|c|c|c|c|c|}
\hline \multicolumn{8}{|c|}{32 VILLAGES DU DISTRICT D’ALLEMBE } \\
\hline \multicolumn{4}{|c|}{16 villages de la zone Yama } & \multicolumn{4}{|c|}{16 villages de la zone Ekassa } \\
\hline \multicolumn{4}{|c|}{128 personnes dans la zone Yama } & \multicolumn{4}{|c|}{128 personnes dans la zone Ekassa } \\
\hline Types de mariage & $\begin{array}{l}\text { Tranche } \\
\text { d'âge des } \\
\text { enquêtés }\end{array}$ & $\begin{array}{l}\text { Profession } \\
\text { des enquêtés }\end{array}$ & $\begin{array}{l}\text { \%des } \\
\text { mariages } \\
\text { selon leur } \\
\text { typologie }\end{array}$ & Types de mariage & $\begin{array}{l}\text { Tranche } \\
\text { d'âge des } \\
\text { enquêtés }\end{array}$ & $\begin{array}{l}\text { Profession } \\
\text { desenquêtés }\end{array}$ & $\begin{array}{l}\% \text { des } \\
\text { mariages } \\
\text { selon leur } \\
\text { typologie }\end{array}$ \\
\hline $\begin{array}{l}\text { 1- Mariage par } \\
\text { anticipation }\end{array}$ & $18-20$ ans & $\begin{array}{l}\text { Sans } \\
\text { profession }\end{array}$ & $0,5 \%$ & $\begin{array}{l}\text { 1-Mariage par } \\
\text { anticipation }\end{array}$ & $18-20$ ans & $\begin{array}{l}\text { Conducteurs } \\
\text { de taxi- } \\
\text { motos }\end{array}$ & $0,8 \%$ \\
\hline 2-Mariage okyénaa & $25-30$ ans & Pêcheurs & $15 \%$ & $\begin{array}{l}\text { 2-Mariage } \\
\text { okyénaa }\end{array}$ & $25-30$ ans & Chasseurs & $10 \%$ \\
\hline 3-Mariage okwélé & $30-40$ ans & Cultivateurs & $25 \%$ & $\begin{array}{l}\text { 3-Mariage } \\
\text { okwélé }\end{array}$ & $30-40$ ans & Malafoutiers & $30 \%$ \\
\hline $\begin{array}{l}\text { 4-Mariage avec la } \\
\text { sœur de l'épouse }\end{array}$ & $40-50$ ans & Malafoutiers & $0,8 \%$ & $\begin{array}{l}\text { 4- Mariage } \\
\text { avec la sœur de } \\
\text { l'épouse }\end{array}$ & $40-50$ ans & $\begin{array}{l}\text { Eleveurs de } \\
\text { moutons }\end{array}$ & $0,2 \%$ \\
\hline $\begin{array}{l}\text { 5-Mariage avec } \\
\text { la fille du frère de } \\
\text { l'épouse }\end{array}$ & $50-60$ ans & Vanniers & $13,5 \%$ & $\begin{array}{l}\text { 5-Mariage avec } \\
\text { la fille du frère } \\
\text { de l'épouse }\end{array}$ & $50-60$ ans & Cultivateurs & $5 \%$ \\
\hline $\begin{array}{l}\text { 6- Polygynie ou } \\
\text { polygamie }\end{array}$ & $60-70$ ans & Retraités & $60 \%$ & $\begin{array}{l}\text { 6- Polygynie ou } \\
\text { polygamie }\end{array}$ & $60-70$ ans & Retraités & $45 \%$ \\
\hline Total & & & $100 \%$ & Total & & & $100 \%$ \\
\hline
\end{tabular}




\section{Références bibliographiques}

Bonte, P. et IzARd, M. (2002) Dictionnaire de l'ethnologie et de l'anthropologie. Paris : PUF.

Bouquet, A. (1969) Féticheurs et médecines traditionnelles du Congo Brazzaville.

Paris : Office de Recherches Scientifiques des Territoires d'Outre-Mer. CNSEE (2009) Annuaire statistique du Congo 2007. Brazzaville : CNSEE (Centre National de la Statistique et des Etudes Economiques).

DAно, E. (1983) Le mariage mbosi : tradition etévolution. Thèse de $3^{\text {ème }}$ cycle de sociologie. Caen : Université de Caen.

Embanga Aborobongui, G. M.; Hamlaoui, F. et Rialland, A. (2014) «Syntactic and prosodic aspects of left and right dislocation in Emb \si (Bantu C25) », Proceedings of the Workshop BantuSynPhonIS : Preverbal Domain(s), $57: 26-48$.

Foullée, A. (1990) La science sociale contemporaine. Paris : Hachette.

Géraud, M.-O.; Lezervoisier, O. et Potier, R. (2000) Les notions clés de l'ethnologie. Paris : Armand Colin.

IтоuA, J. (2007) Les mbosi au Congo : peuple et civilisation. Paris : L'Harmattan. SAUTTER, G. (1962) La cuvette congolaise. Paris : Servant Crouzet.

Soret, M. (1954) Démographie et problèmes urbains en A.E.F. Mémoires de l'Institut d'Etudes Centrafricaines, 7.

Soussa, L. (1981) Evolution des structures sociales traditionnelles au Congo : de la pénétration coloniale française à nos jours : exemple mbochi. Thèse de $3^{\text {ème }}$ cycle d'études africaines. Paris : Ecole des hautes Etudes en Sciences Sociales.

SPIRO, M. (1975) «Marriage payments: a paradigm from the burmese perspective », Journal of anthropological research, 31: 89-115.

TAmbiah, S. et Goody, J. (1973) Bridewealth and dowry. Cambridge: Cambridge University Press.

Zambo Belinga, J.-M. (2003) «Quête de notabilité sociale, rémanence autoritaire et démocratisation au Cameroun », Cahiers d'études africaines, $171: 573-589$. 


\section{Glossaire}

Omvè : segment de lignage, lignée.

Asia : lignage.

Baré bà omwè ophoo: membres d'un même lignage.

Aboro alongo, aboro l'ékoma: la parenté c'est le lien du sang.

Moro onè o baré : l'homme est grand grâce aux gens.

Oyiri wo la abora: Cette femme possède des maternités.

Ebandi ou mbandi : choisir, élire.

Dzémi: grossesse.

$\mathrm{Nzi}$ : monnaie en coquillage.

Ngyèlè : trois barrettes servant de monnaie ancienne

Ikyéna : couper, sectionner.

Okyénaa : union matrimoniale issue d'une rupture provoquée par une femme mariée bénéficiant de la complicité de son nouveau partenaire. Ipigni wa mwasi : je lui ai ravi sa femme.

okwélé vient du radical

Kwélé : Biens, humains et matériels, laissés par le défunt.

Owé : Défunt.

Okwélé : veuvage, deuil.

Ayiri ba kwélé : les veuves

oyiri ya kwélé : la veuve

Abaa ba kwélé : les veufs

Bana ba abola : neveux utérins.

Asombi oyiri ya kwélé : acheter la veuve.

Lémbindi, mbindi : souillure.

Ngwanga : servitude.

$\mathrm{Ngo0}$ : la mère

$i b a$, «le mariage »;

Iba alongi : mariage réussi.

Obwè : dot

Tasamwaré : tante maternelle 